\title{
Wall Effect Analysis in Thermal-Hydraulics Aspect of HTR-10 and PR-3000 Reactors Using Different Porosity Models
}

\author{
Bilal El Bari ${ }^{1}$, Dwi Irwanto ${ }^{2 *}$ \\ ${ }^{1}$ Departement of Physics, Faculty of Mathematics and Natural Sciences, Institut Teknologi Bandung, Bandung, 40132, Indonesia \\ ${ }^{2}$ Nuclear Physics and Biophysics Research Group, Department of Physics, Faculty of Mathematics and Natural Sciences, Institut Teknologi Bandung, Bandung, 40132, Indonesia \\ *Corresponding author: dirwanto@fi.itb.ac.id
}

\begin{abstract}
Gen-IV is the latest generation of a nuclear reactor with better aspects than the previous generation, Pebble-Bed Reactor (PBR) is one of them. PBR using spherical shape fuel or known as pebble fuel. Hence, the physical things that are happening in the core are significantly different from the reactor using a fuel pin. Often the reactor core is assumed as a porous medium because of the void within the pebble fuel. The coolant that moves in the core can be carried as a fluid that flows through a porous medium. The thermal-hydraulics aspect is one of the significant aspects that must have been considered in every nuclear fission reactor because it is closely related to reactor safety. The wall effect happened in a packed bed, making the porosity near-wall position higher than other positions. The porosity value can induce the coolant flow, known as wall channeling, which can influence the reactor's thermal-hydraulics aspect. In this study, thermal-hydraulics analysis for PBR is performed, and the wall effect is taken into account in the calculations. A thermal-hydraulic code, namely mPEBBLE, was developed based on the PEBBLE code to include the wall effect in the calculation. The code is based on the finite-difference method with axisymmetric cylinder geometry $(\mathrm{R}-\mathrm{Z})$ that consists of 4 primary equations; stream flow, pressure recovery, solid energy balance, and thermal energy balance. The analysis was performed for HTR-10 and PR-3000, two PBR-type reactors with different reactor core sizes. The results conclude that the larger bed as in PR-3000 is undergoing a smaller effect caused by the wall effect than in the smaller bed, such as the HTR-10. Comparison of three porosity models, Vortmeyer-Schuster, Mueller, and Benenati-Borislow, to the wall channeling, were also taken into account in the present study.
\end{abstract}

Keywords

Pebble Bed Reactor, Porosity Distribution Model, Thermal-Hydraulic, Temperature

Received: 29 March 2021, Accepted: 23 July 2021

https://doi.org/10.26554/sti.2021.6.4.235-241

\section{INTRODUCTION}

Nuclear fission reactors strived to be better from time to time. The latest generation of the reactor, known as Gen-IV, has better characteristics than the previous generation; safe, secure, sustainable, competitive, and versatile (NEA, 2014). PebbleBed Reactor (PBR) is classified into Very-High Temperature Reactor (VHTR), a Gen-IV type reactor, PBR can produce the high-temperature output.

Thermal-hydraulic and safety aspects are very important factors in PBR, especially considering the unique character of its design. Therefore many studies have been performed to investigate thermal-hydraulic parameters (Zheng et al., 2012; Irwanto et al., 2017), safety and accident analysis (Reitsma, 2012; Irwanto and Obara, 2013), including computation methods of PBR design (Rodríguez et al., 2021; van Rensburg and Kleingeld, 2011).
HTR-10 is one of the PBR types that operate on low power, around $10 \mathrm{MWth}$. This reactor was built by the Institute of Nuclear Energy Technology (INET) (Jing et al., 2002; Wang et al., 2014). There are two German fuel types; conventional and shell, HTR-10 using conventional German type fuel, and this reactor using multi-pass for fuel load scheme, or the fuel enters the core several times. The active core dimension is relatively small; the diameter is $1.8 \mathrm{~m}$, and the height is 1.875 m. HTR-10 cross-section is shown in Figure 1.

PR-3000 is another PBR type that was designed by Kernforschungsanlage Jülich $\mathrm{GmbH}$ that has never been built. This reactor is meant to be the study case to know how the PBR reactor performs on the commercial-scale because PR-3000 has a high operation power of around 3000 MWth. PR-3000 using German fuel. However, there are no particular types, whether conventional or type, because this reactor is just a concept. 


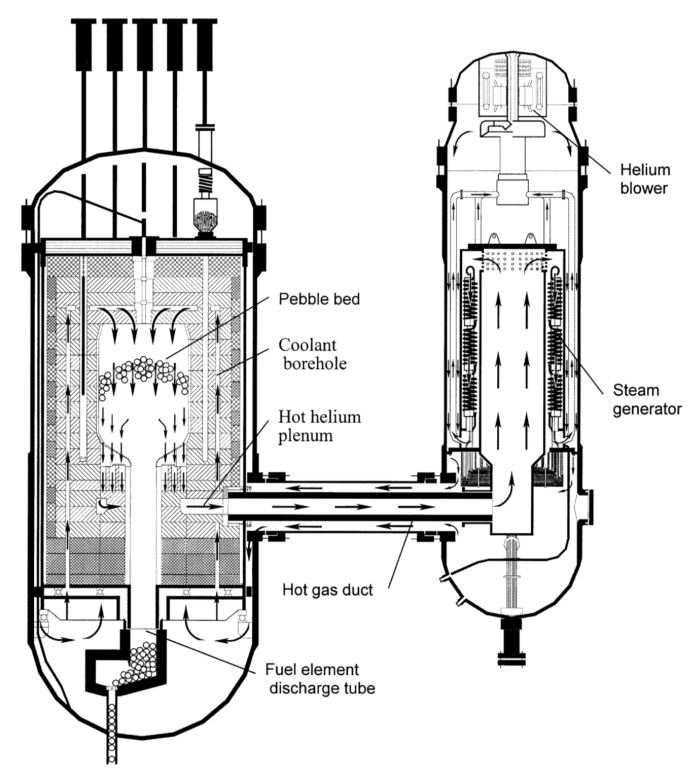

Figure 1. HTR-10 cross-section (Gao and Shi, 2002)

Unlike HTR-10, PR-3000 using the One-Trough-Then-Out (OTTO) fuel scheme. It means that the fuel is inserted into the core and taken out when used. Because PR-3000 has high output power, it has a larger active core dimension than HTR10; the diameter is $9.22 \mathrm{~m}$ and height of $5 \mathrm{~m}$ (Stroh et al., 1979). PR-3000 cross-section is shown in Figure 2.

Because the PBR reactors use spherical fuel filling the core randomly, the safety analysis and thermal-hydraulics analysis are different from the conventional reactors that use fuel assembly. For the thermal-hydraulics analysis in PBR, many studies are modeling the core with the porous medium to know the fluid flow behavior inside the core; because of this, Darcy's law is often used as a base, like the studies in the HTR-10 reactor that has been done by Turkey, China, Korea in the report that entitled Evaluation of High Temperature Gas Cooled Reactor Performance: Benchmark Analysis Related to the PBMR-400, PBMM, GT-MHR, HTR-10 and the ASTRA Critical Facility (Tobergte and Curtis, 2013). For the PR-3000, because the reactor itself is a concept reactor, the thermal-hydraulics analysis was only done by a paper and a report that uses the same concept by KFA Jülich (Stroh et al., 1979).

Forchheimer equation is Darcy's law that has been modified. This equation is often used in fluid dynamics on a porous medium that has relatively fast flow. The form of the Forchheimer equation that frequently used is the semi-empirical Ergun equation,

$$
\frac{\Delta P}{x}=\frac{1-\phi}{\phi^{3}}\left[150(1-\phi) \frac{\mu v}{d_{p}}+1.75 \frac{\rho}{d_{p}} v^{2}\right]
$$

where $\mathrm{P}$ is the pressure, $\mathrm{x}$ is the position, $\phi$ is the bed void fraction, $\mathrm{v}$ is the superficial velocity, $\mu$ is the fluid viscosity, $\rho$

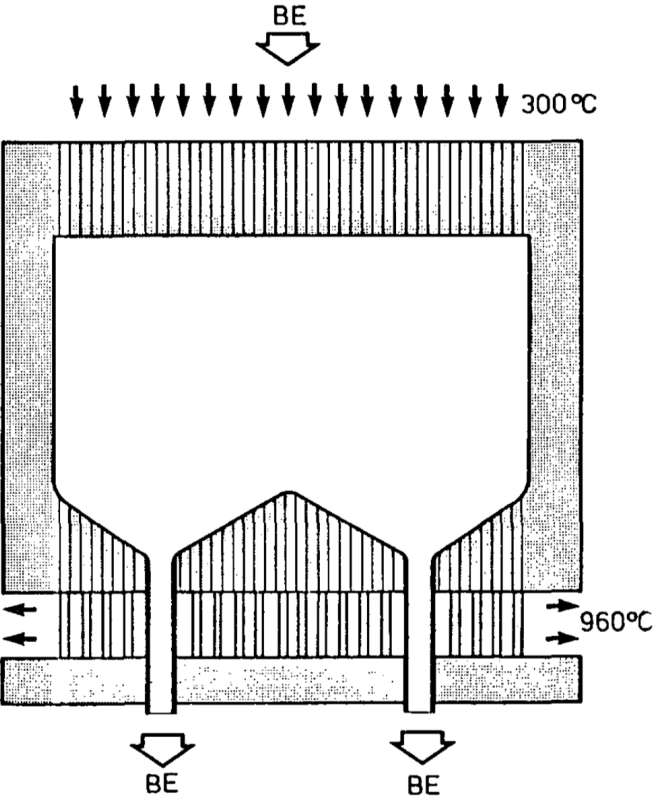

Figure 2. PR-3000 cross-section (Stroh et al., 1979)

is the fluid density, and $\mathrm{d}_{p}$ is the pebble-fuel diameter (Stroh et al., 1979).

Commonly for studies in fluid flow through the packedbed system, the value of porosity has assumed homogenous (uniform void value in all positions). The research was done by Benenati and Brosilow (1962), points out that the porosity value near the wall is increasing. It happens because the contact between the pebble and wall makes the void larger than in another position. Because the porosity near the wall is enormous, it makes the fluids tend to flow through larger porosity. The phenomenon is called wall channeling (Al Falahi et al., 2018; Du Toit, 2008).

The present study aims to analyze the wall channeling effect on the Pebble Bed Reactor's thermal-hydraulic aspect using different porosity models, namely Benenati-Borislow, Mueller, and Vortmeyer-Schuster. The wall effect is taken into account by modifying the PEBBLE code to include the porosity models in the calculation. Analysis for different reactor core sizes, HTR-10 and PR-3000, are also performed in the present study to investigate the effect of reactor core geometry on the wall channeling.

\section{EXPERIMENTAL SECTION}

\subsection{Governing Equations}

PEBBLE is the program that calculates the thermal-hydraulics aspect of PBR type, and Kenneth R. Stroh made it in 1979 to review the thermal-hydraulics aspect of PR-3000 and written in FORTRAN language (Stroh et al., 1979). The PEBBLE calculation method's foundation is finite-difference with axisymmetric cylinder geometry (R-Z) that consists of 4 primary equations; stream flow, pressure recovery, solid energy balance, 
and thermal energy balance.

mPEBBLE is a program developed based on the PEBBLE code. The most apparent thing about mPEBBLE than the PEBBLE is that it can consider the heterogeneous porosity distribution on the calculation. The porosity value is inputted in the csv file, a 2 -dimensional array (representing $\mathrm{R}-\mathrm{Z}$ position). However, active core porosity can also be made as a homogenous region if the user inputs the same value for all positions.

The three models are used for the porosity distribution model; equation from Benenati-Borislow experiment, Vort meyer-Schuster porosity distribution model, and Mueller porosity distribution model. For the Benenati-Borislow experiment equation, the porosity distribution is divided into two values; the core and near-wall regions (Benenati and Brosilow, 1962). The near-wall area occupies half of the pebble diameter, $3 \mathrm{~cm}$ from the wall, and the rest of it is the core region. The value of porosity in the core region is represented by

$$
\phi_{c}=\frac{\phi_{b}-\phi_{w}}{\left(1-\frac{d_{p}}{D}\right)^{2}}+\phi_{w}
$$

and for the near-wall region

$$
\phi_{w}=\frac{63.6}{\left(\frac{D}{d_{p}}+15\right)^{2}}+0.43
$$

Where $\phi_{c}$ is the porosity value of the core region, $\phi_{w}$ is the porosity value of the near-wall region, $\phi_{b}$ is the bulk porosity, and the value is 0.39 for HTR-10 or PR-3000, and D is the bed diameter.

The Vortmeyer-Schuster porosity distribution model does not represent the actual porosity value at a certain point, but the model represents the average porosity distribution. The model is described in the exponential equation,

$$
\phi(r)=1-\left(1-\phi_{b}\right)\left\{1-e^{-2\left(\frac{R-r}{d_{p}}\right)^{2}}\right\}
$$

Where $\mathrm{R}$ is the radius of bed and $\mathrm{r}$ is the observation position, the value of porosity in the Vortmeyer-Schuster model is never decreased, which means porosity value near-wall is the highest, and gradually the value is falling by the relative position to a wall, and the minimum value is located in center bed (Al Falahi et al., 2018).

Mueller's porosity distribution model is the model that represents the actual value of porosity in packed-bed. The model is represented by the equation, which like a damped oscillation as

if $\mathrm{D} / \mathrm{d} \geq 2.02$

$$
\phi(r *)=\phi_{b l}+\left(1+\phi_{b l}\right) J_{0}(a r *) e^{b r *}
$$

with

$$
\begin{aligned}
& \text { if } 2.02 \geq D / d_{p} \geq 13.0 a=7.45-\frac{3.15}{D / d_{p}} \\
& \text { if } \mathrm{D} / \mathrm{d}_{p} \leq 13.0 \\
& a=7.45-\frac{11.25}{D / d_{p}} \\
& b=0.315-\frac{0.725}{D / d_{p}} \\
& \text { if } \mathrm{r} / \mathrm{d}_{p} \geq 0 \\
& \mathrm{r}^{*}=r / d_{p}
\end{aligned}
$$

and

$$
\phi_{b l}=0.365+\frac{0.220}{D / d_{p}}
$$

where J0 is the zero-order Bessel function (Mueller, 1992). For simplicity, the porosity value input for Mueller model observation is not all of the positions are represented by the value from equation (5). Still, only the region within half of the wave crest near-wall region is represented.

\subsection{Calculation Methods}

Three types of calculations were performed for the HTR-10 thermal-hydraulics aspect based on three different porosity models: Benenati-Borislow, Mueller, and Vortmeyer-Schuster. The results will be compared to the FLUENT program's result using the Benenati-Borislow porosity model performed by Hacettepe University, Turkey (Tobergte and Curtis, 2013). Figure 3 shows the HTR-10 cores porosity values assigned to the mPEBBLE program, which used different porosity models.

For PR-3000 calculation, three different porosity models, Benenati-Borislow, Mueller, and Vortmeyer-Schuster are also be used. The calculation results will also be compared to the homogenous porosity model to investigate the effects of each porosity model. Figure 4 shows the PR-3000 cores porosity values assigned to the mPEBBLE program, which used a different porosity model.

The core power density for HTR-10 is obtained from HTR10 benchmarks performed by the Institute of Nuclear and New Energy Technology (INET) in the report with the title of "Evaluation of High-Temperature Gas-Cooled Reactor Performance: Benchmark Analysis Related to the PBMR-400, PBMM, GT-MHR, HTR-10, and the ASTRA Critical Facility" (Tobergte and Curtis, 2013). The interpolated is shown in Figure 5.

For PR-3000, the power density data is obtained from the report with the title "Thermal-Hydraulic Analysis Techniques for Axisymmetric Pebble Bed Nuclear Reactors" (Stroh et al., 1979). A more specified power density data have been interpolated, as shown in Figure 6. 

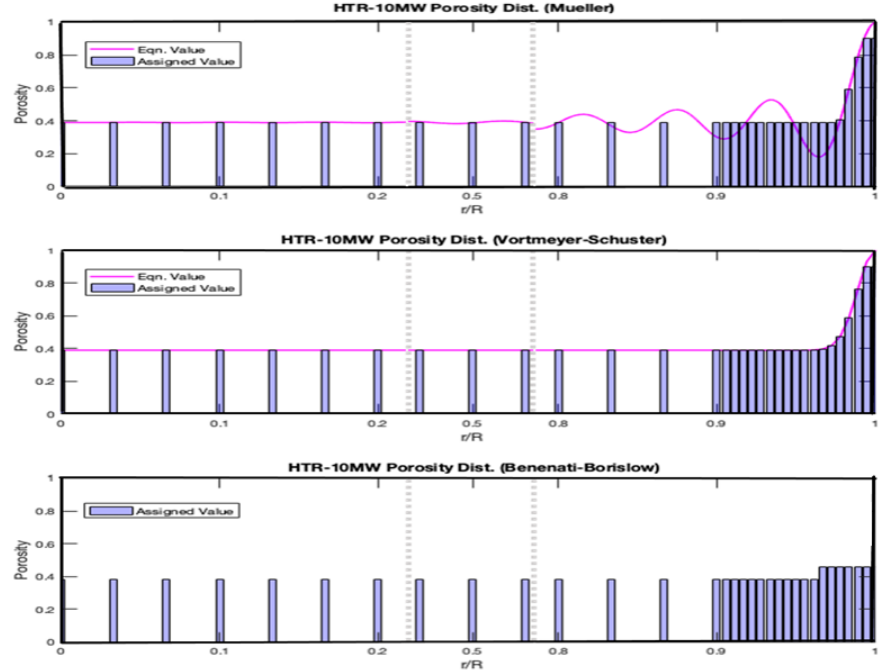

Figure 3. Three Different Porosity Models in HTR-10
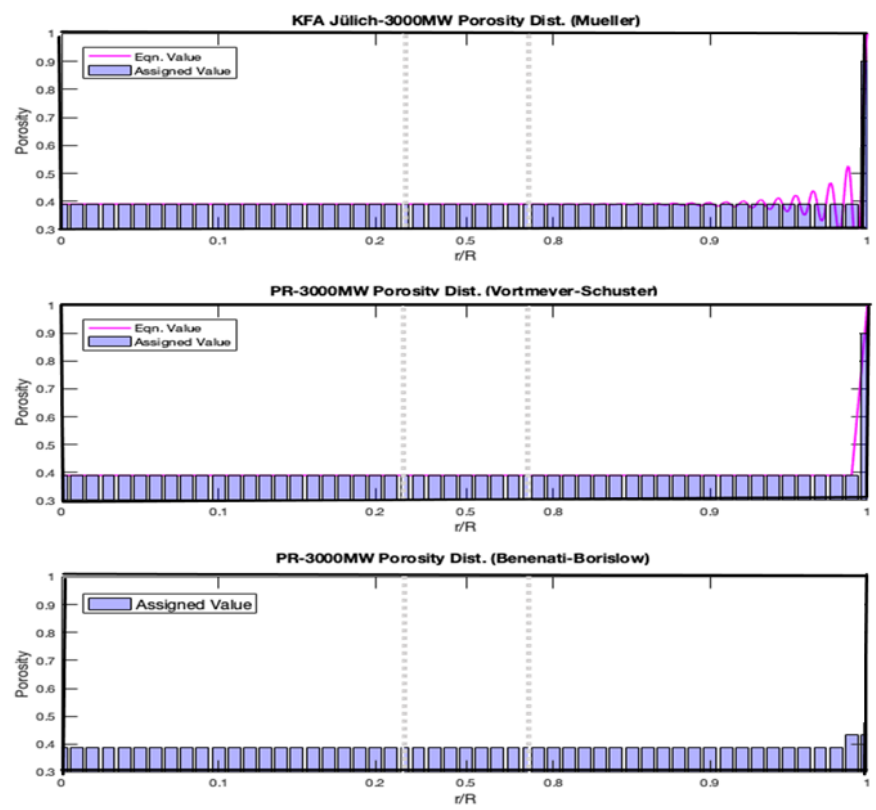

Figure 4. Three Different Porosity Models in PR-3000

\section{RESULTS AND DISCUSSION}

\subsection{HTR-10 Calculation}

Figure 7 shows coolant outlet temperature distribution comparison using mPEBBLE for different pososity models, BenenatiBorislow Mueller and Vortmeyer-Schuster. Figures 8, 9, 10 shows coolant temperature distribution calculated using mPEBBLE for Benenati-Borislow, Mueller, and Vortmeyer-Schuster porosity distribution, respectively. Each figure shows temperature in three constant axial and radial positions for HTR10 and its comparison with FLUENT calculation result using Benenati-Borislow model (Tobergte and Curtis, 2013).

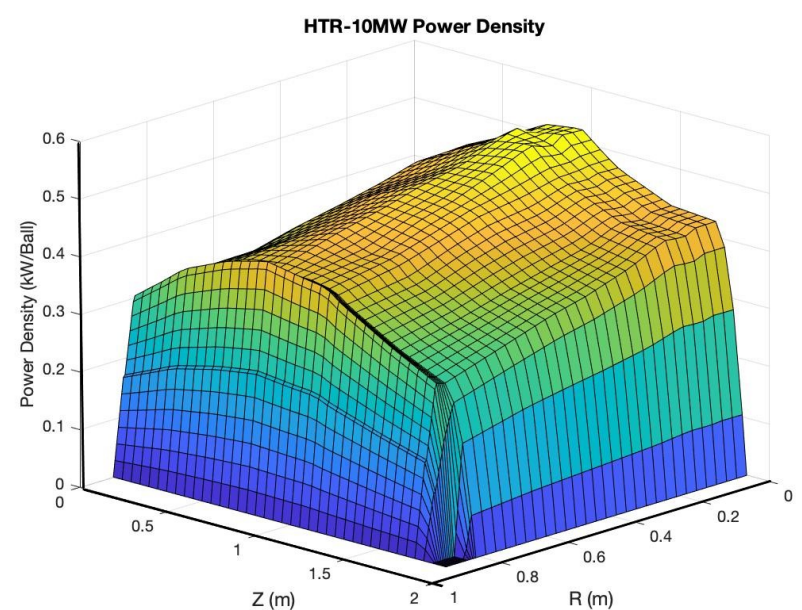

Figure 5. HTR-10 Power Density Profile

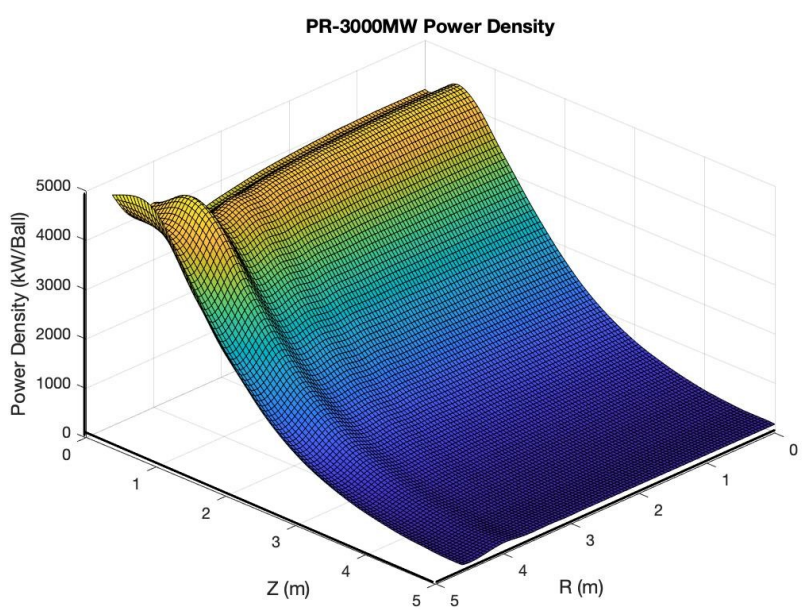

Figure 6. PR-3000 Power Density Profile

Figure 8 shows coolant temperature distribution comparison between FLUENT and mPEBBLE using the same porosity model (Benenati-Borislow). Figure 9 shows coolant temperature distribution comparison between FLUENT and mPEBBLE using different porosity models (FLUENT using BenenatiBorislow, while mPEBBLE using Mueller model). Figure 10 shows coolant temperature distribution comparison between FLUENT and mPEBBLE using different porosity models (FLUENT using Benenati-Borislow, while mPEBBLE using Vortmeyer-Schuster model).

Calculation using the FLUENT and Benenati-Borislow model is used as a reference in the present study due to its data availability. Temperature distribution calculated using mPEBBLE and Benenati-Borislow relatively has the same results compared to FLUENT and Benenati-Borislow model. This calculation could be used as a benchmark calculation to 


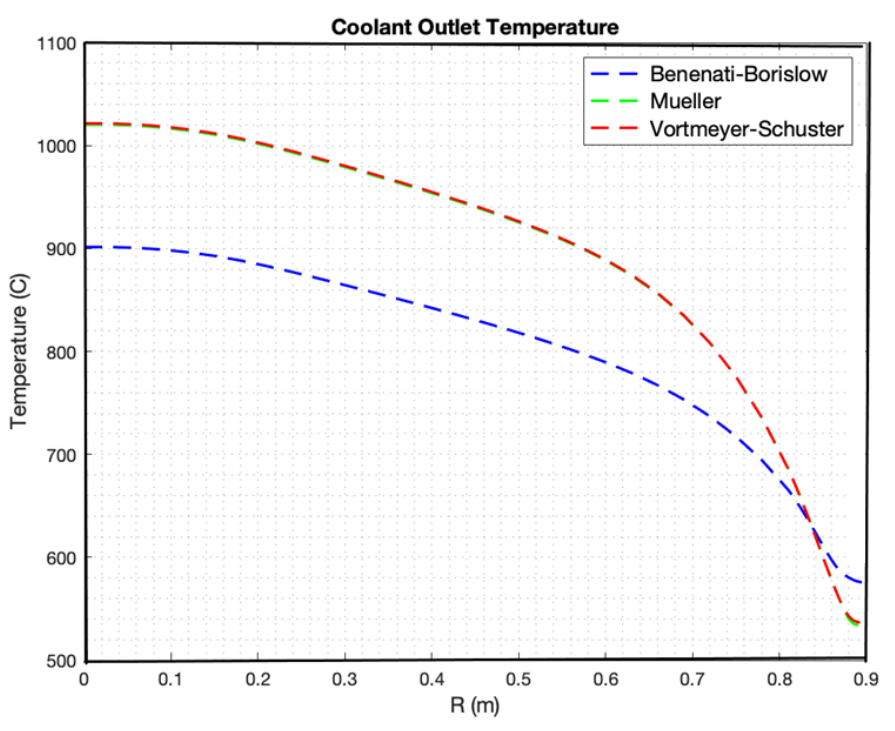

Figure 7. Coolant Outlet Temperature Distribution Comparison mPEBBLE (Benenati-Borislow)[Calc.] mPEBBLE (Mueller)[Calc.] - mPEBBLE

(Vortmeyer-Schuster)[Calc.]

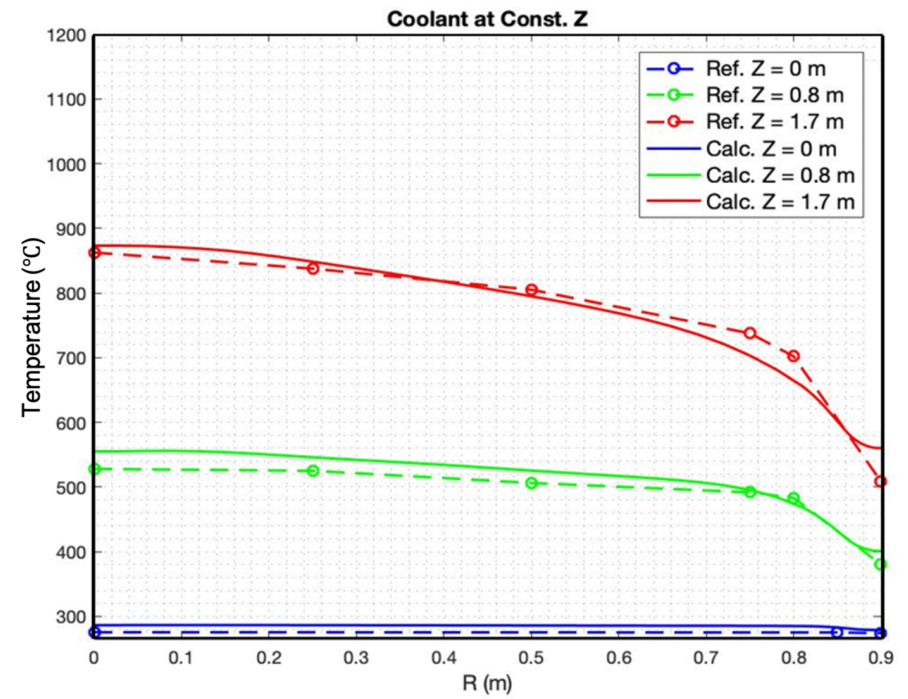

Figure 8. Coolant Temperature Distribution Comparison FLUENT(Benenati-Borislow)[Ref.] - mPEBBLE (Benenati-Borislow)[Calc.]

prove that the numerical methods implemented in mPEBBLE are correct and verified. The next task is to calculate using different porosity models, the Vortmeyer-Schuster model, and Mueller models, as shown in Figure 8 and 9. The different nature of the numerical model and boundary condition for computational fluid dynamics using finite volume method use in FLUENT and finite different model use in mPEBBLE could be contributed to these differences. Value assignment of the reactor's power density at the wall could also be one of the

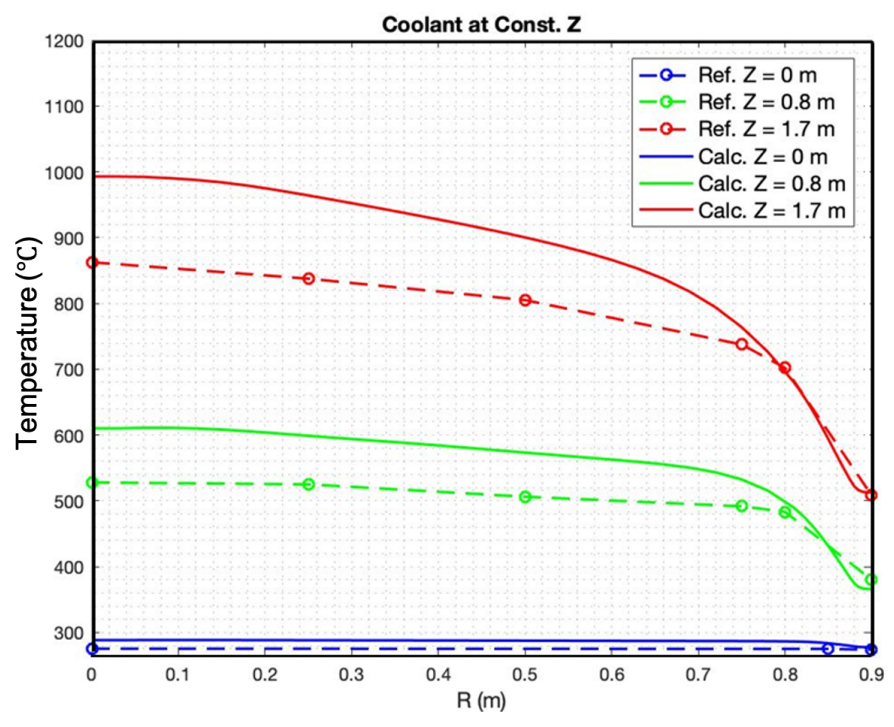

Figure 9. Coolant Temperature Distribution Comparison FLUENT(Benenati-Borislow)[Ref.] - mPEBBLE (Mueller)[Calc.]

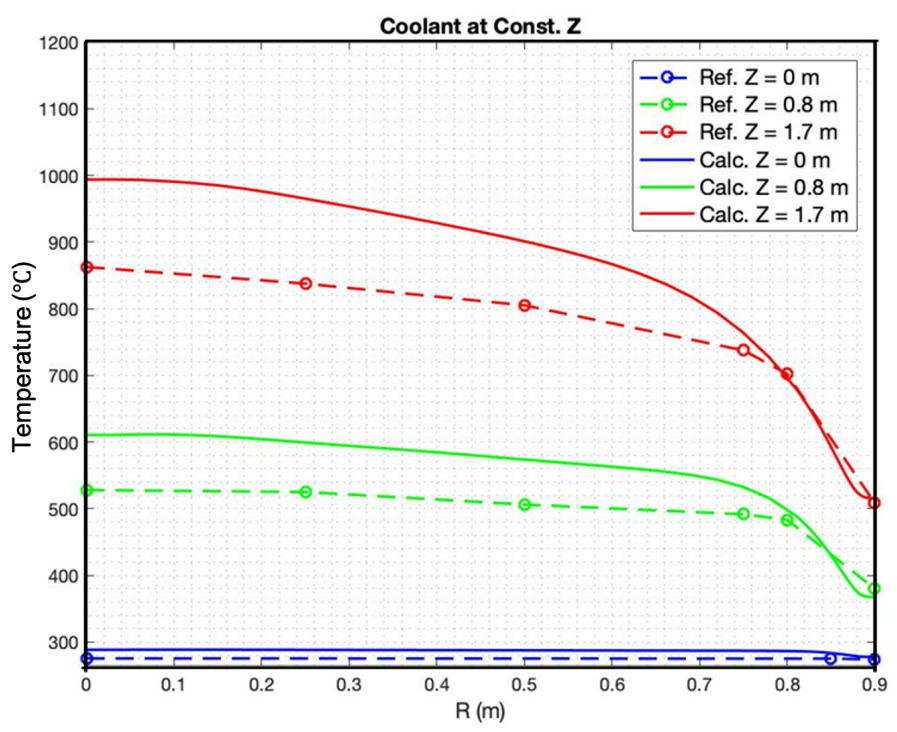

Figure 10. Coolant Temperature Distribution Comparison FLUENT(Benenati-Borislow)[Ref.] - mPEBBLE (Vortmeyer-Schuster)[Calc.]

reasons, as the finite volume method uses cell-centered formulation. Moreover, the number of grid/points and the porosity value assigned in every point also contribute to the different results between FLUENT and MPEBBLE.

The calculation results in mPEBBLE using the Mueller or Vortmeyer-Schuster porosity model have identical results. However, the Vortmeyer-Schuster porosity model has a slightly higher temperature in the near-wall position, with the difference is around $2^{\circ} \mathrm{C}$. The temperature difference between these two porosity models is small. It happens because the porosity 


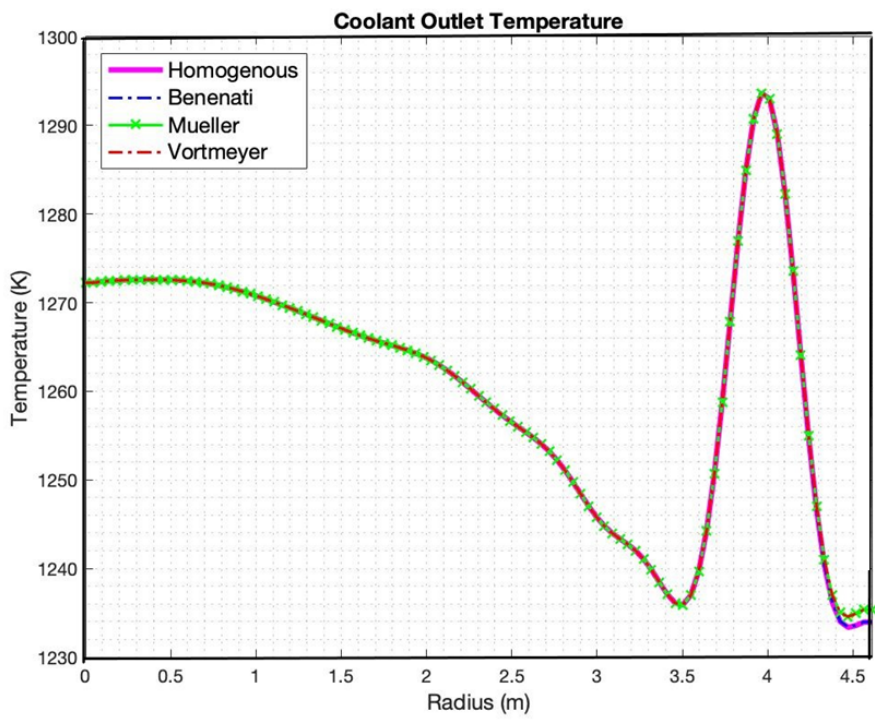

Figure 11. Coolant Outlet Temperature Distribution in Mpebble Using Four Different Porosity Model; Homogenous, Benenati-Borislow, Mueller, and Vortmeyer-Schuster

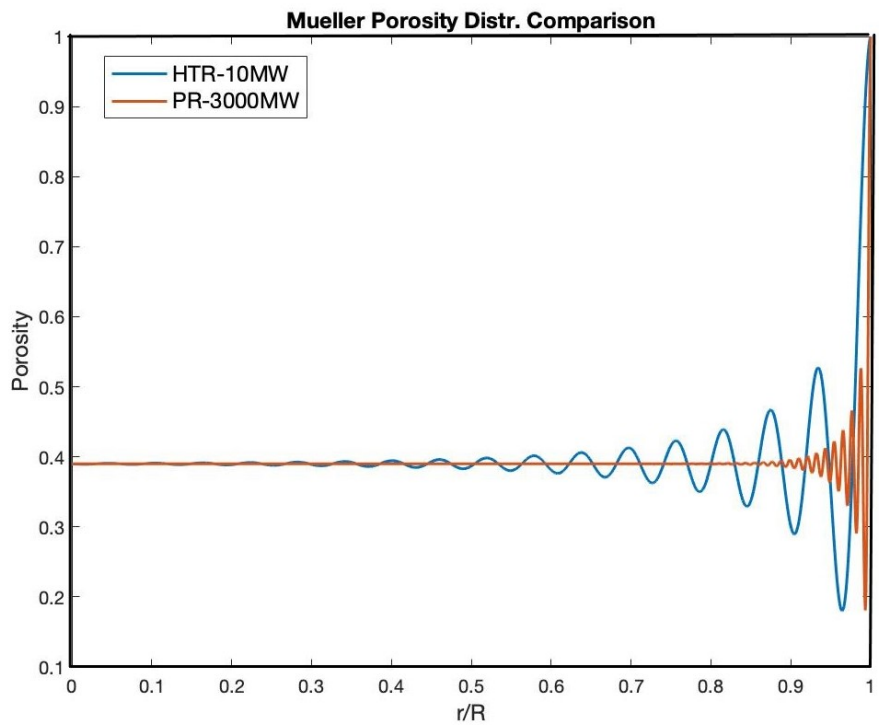

Figure 12. Mueller Porosity Distribution in HTR-10 and PR-3000 on A Dimensionless Radius

values assigned are quite the same, as shown in Figure 3. Compared to the FLUENT result that uses the Benenati-Borislow porosity model, these two models have a noticeable profile because the porosity in Mueller and Vortmeyer-Schuster model is higher than the Benenati-Borislow model in the near-wall position, as shown in Figure 3. Different porosity at the nearwall region makes the wall channeling effect more noticeable in the Mueller and Vortmeyer-Schuster model than that of the Benenati-Borislow porosity model. The more apparent wall channeling effect is inducing lower temperature in the near-

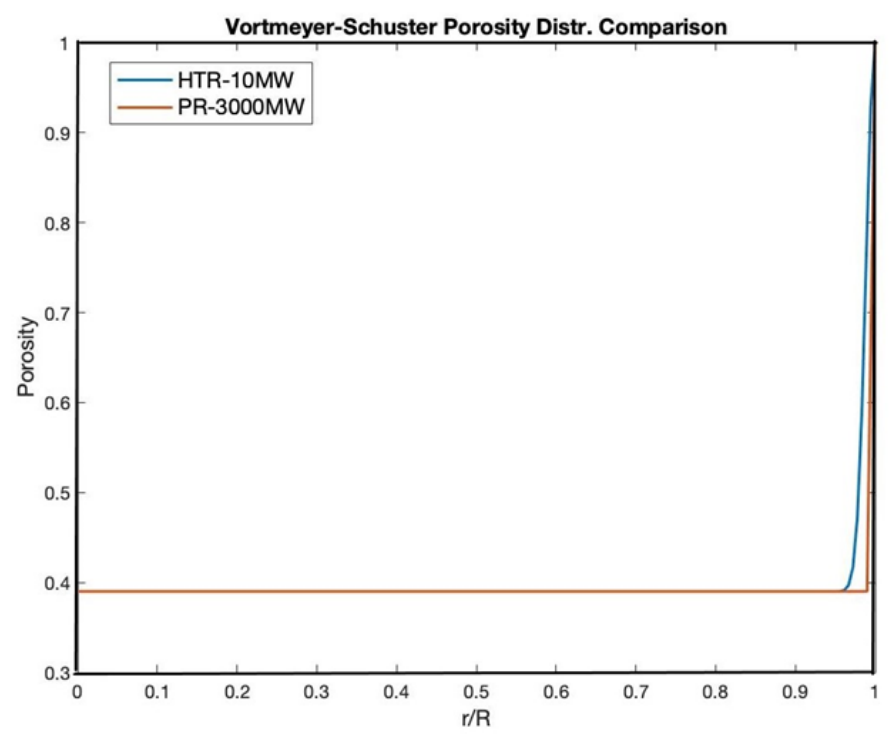

Figure 13. Vortmeyer-Schuster Porosity Distribution in HTR-10 and PR-3000 on A Dimensionless Radius

wall position and higher temperature in the core position due to the mass flow in the near-wall position is higher than in other positions. As the result, there is quite a significant maximum temperature difference at the center of the core, at around $130^{\circ} \mathrm{C}$, between Mueller and Vortmeyer-Schuster model and the Benenati-Borislow porosity model.

\subsection{PR-3000 Calculation}

Analysis of different porosity models continued for different reactor core sizes, as the reactor core diameter has a significant effect, both in the neutronic and thermal-hydraulic aspects of the reactor. Figure 11 compares the coolant temperature results in the radial direction for the PR-3000 reactor using four different porosity distribution models, Homogenous, BenenatiBorislow, Mueller, and Vortmeyer-Schuster porosity model.

From Figure 11, it can be seen that the differences between the four different models are not significant in the PR-3000 reactor. The results obtained from neither the Mueller model nor the Vortmeyer-Schuster model have no significant effect as it happened in the HTR-10 calculation. Compared to the HTR-10, Mueller and Vortmeyer-Schuster porosity models applied in PR-3000 have no steep increase at the near-wall core position, as shown in Figures 12 and 13. Both Mueller and Vortmeyer-Schuster porosity models in PR-3000 calculation have almost the same value at the near-wall of the core that produced nearly the same temperature distribution. Compared to the homogenous and Benenati-Borislow porosity model, the maximum temperature difference between the Mueller or Vortmeyer-Schuster is pretty small at around $1^{\circ} \mathrm{C}$.

In PR-3000, the temperature differences between different porosity models are slight because the particle and bed diameter influence the wall channeling effect. The bigger bed diameter makes the wall channeling effect small because the bed can be 
treated more like a homogenous bed. Compared to PR-3000, HTR-10 has the same particle size but a smaller bed, making the wall channeling effect in HTR-10 more significant.

Compared to FLUENT, mPEBBLE has dynamic porosity distribution inputs so that the wall channeling effect can be observed. mPEBBLE is a deterministic code that could be run faster than Computational Fluid Dynamic code such as FLUENT, depending on the meshing. Moreover, one can access the mPEBBLE source's code to be easier for direct coupling with other nuclear reactor code, such as the neutronic or safety aspect of the reactor.

\section{CONCLUSIONS}

The wall effect analysis in the thermal-hydraulics aspect of HTR-10 and PR-3000 reactor has been investigated using the mPEBBLE code, a modified version of PEBBLE code, by considering different porosity models: Benenati-Borislow, Mueller, and Vortmeyer-Schuster porosity models. From the calculation, it is found that for the HTR-10 reactor, the BenenatiBorislow porosity model has a significant difference from the Mueller or Vortmeyer-Schuster porosity models, especially at the near wall of the core that leads to the temperature difference at the other region of the reactor. As for the PR-3000 reactor, Benenati-Borislow, Mueller, and Vortmeyer-Schuster porosity models have no significant effect on the temperature distribution along with the reactor core. It is found that the size of the reactor significantly affects the application of the porosity models at the near-wall region and its temperature distribution. A small reactor core such as HTR-10 has a significant near-wall effect, while a larger rector core size of PR-3000 is not significantly affected by the near-wall effect.

\section{ACKNOWLEDGEMENT}

This research was supported the Indonesia's Ministry of Research Technology and Higher Education, through the University's Excellence Applied Research (PUPT) Grant and Bandung Institute of Technology through the Research and Innovation ITB Grant.

\section{REFERENCES}

Al Falahi, F., G. Mueller, and M. Al-Dahhan (2018). Pebble bed nuclear reactor structure study: A comparison of the experimental and calculated void fraction distribution. Progress in Nuclear Energy, 106; 153-161

Benenati, R. and C. Brosilow (1962). Void fraction distribution in beds of spheres. AIChE Journal, 8(3); 359-361

Du Toit, C. G. (2008). Radial variation in porosity in annular packed beds. Nuclear engineering and design, 238(11); 30733079
Gao, Z. and L. Shi (2002). Thermal hydraulic calculation of the HTR-10 for the initial and equilibrium core. Nuclear Engineering and Design, 218(3); 51-64

Irwanto, D. and T. Obara (2013). Decay heat removal without forced cooling on a small simplified PBR with an accumulative fuel loading scheme. Annals of Nuclear Energy, 60; 383-395

Irwanto, D., T. Setiadipura, and A. Pramutadi (2017). Comparison of several thermal conductivity constants for thermal hydraulic calculation of pebble bed reactor. IOP Publishing, 877(1); 012031

Jing, X., X. Xu, Y. Yang, and R. Qu (2002). Prediction calculations and experiments for the first criticality of the 10 MW High Temperature Gas-cooled Reactor-Test Module. Nuclear Engineering and Design, 218(3); 43-49

Mueller, G. E. (1992). Radial void fraction distributions in randomly packed fixed beds of uniformly sized spheres in cylindrical containers. Powder technology, 72(3); 269-275

NEA, O. (2014). Technology Roadmap Update for Generation IV Nuclear Energy Systems. OECD

Reitsma, F. (2012). Reactivity considerations for the on-line refuelling of a pebble bed modular reactor-Illustrating safety for the most reactive core fuel load. Nuclear Engineering and Design, 251; 18-29

Rodríguez, A. G., L. Y. R. Mazaira, C. R. G. Hernández, D. S. Dominguez, and C. A. B. de Oliveira Lira (2021). An integral 3D full-scale steady-state thermohydraulic calculation of the high temperature pebble bed gas-cooled reactor HTR10. Nuclear Engineering and Design, 373; 111011

Stroh, K., R. Jiacoletti, and H. Olson (1979). Thermalhydraulic analysis techniques for axisymmetric pebble bed reactor cores. Nuclear Engineering and Design, 52(3); 343347

Tobergte, D. R. and S. Curtis (2013). Evaluation of High Temperature Gas Cooled Reactor Performance: Benchmark Analysis Related to the PBMR-400, PBMM, GT-MHR, HTR-10 and the ASTRA Critical Facility. International Atomic Energy Agency, 53(9); 1-690

van Rensburg, J. J. and M. Kleingeld (2011). An integral CFD approach for the thermal simulation of the PBMR Reactor Unit. Nuclear engineering and design, 241(8); 3130-3141

Wang, M.-J., R.-J. Sheu, J.-J. Peir, and J.-H. Liang (2014). Criticality calculations of the HTR-10 pebble-bed reactor with SCALE6/CSAS6 and MCNP5. Annals of Nuclear Energy, 64; 1-7

Zheng, Y., J. Lapins, E. Laurien, L. Shi, and Z. Zhang (2012). Thermal hydraulic analysis of a pebble-bed modular high temperature gas-cooled reactor with ATTICA3D and THERMIX codes. Nuclear Engineering and Design, 246; 286-297 